\title{
Laparoscopic versus open sigmoid resection for diverticular disease: follow-up assessment of the randomized control Sigma trial
}

\author{
Bastiaan R. Klarenbeek - Roberto Bergamaschi - Alexander A. F. A. Veenhof • \\ Donald L. van der Peet • Wim T. van den Broek • Elly S. M. de Lange • \\ Willem A. Bemelman • Pieter Heres • Antonio M. Lacy • Miguel A. Cuesta
}

Received: 3 June 2010/ Accepted: 7 August 2010/Published online: 25 September 2010

(C) Springer Science+Business Media, LLC 2010

\begin{abstract}
Background The short-term results of the Sigma trial show that laparoscopic sigmoid resection (LSR) used electively for diverticular disease offers advantages over open sigmoid resection (OSR). This study aimed to compare the overall mortality and morbidity rates after evaluation of the clinical outcomes at the 6-month follow-up evaluation.

Methods In a prospective, multicenter, double-blind, parallel-arm, randomized control trial, eligible patients were randomized to either LSR or OSR. The short-term results and methodologic details have been published previously. Follow-up evaluation was performed at the outpatient clinic 6 weeks and 6 months after surgery.

Results In this trial, 104 patients were randomized for either LSR or OSR, and the conversion rate was $19.2 \%$. The LSR approach was associated with short-term benefits such as a $15.4 \%$ reduction in the major complications rate,
\end{abstract}

Presented at the 12th WCES, April 14-17, 2010, National Harbor, MD.

B. R. Klarenbeek · A. A. F. A. Veenhof .

D. L. van der Peet . W. T. van den Broek .

E. S. M. de Lange $\cdot$ M. A. Cuesta ( $\square)$

Department of Surgery, VU University Medical Center,

De Boelelaan 1117, 1081 HV Amsterdam, The Netherlands

e-mail:ma.cuesta@vumc.nl

R. Bergamaschi

Forde Health System, Bergen University, Forde, Norway

W. A. Bemelman

Academic Medical Center, Amsterdam, The Netherlands

P. Heres

Waterland Hospital, Purmerend, The Netherlands

A. M. Lacy

Hospital Clinic, Barcelona, Spain less pain, and a shorter hospital stay at the cost of a longer operating time. At the 6-month follow-up evaluation, no significant differences in morbidity or mortality rates were found. Two patients died of cardiac causes (overall mortality, 3\%). Late complications (7 LSR vs. 12 OSR; $p=$ $0.205)$ consisted of three incisional hernias, five small bowel obstructions, four enterocutaneous fistulas, one intraabdominal abscess, one retained gauze, two anastomotic strictures, and three recurrent episodes of diverticulitis. Nine of these patients underwent additional surgical interventions. Consideration of the major morbidity over the total follow-up period (0-6 months) shows that the LSR patients experienced significantly fewer complications than the OSR patients (9 LSR vs. 23 OSR; $p=0.003$ ). The Short Form-36 (SF-36) questionnaire showed significantly better quality of life for LSR at the 6-week follow-up assessment. However, at the 6-month follow-up assessment, these differences were decreased.

Conclusions The late clinical outcomes did not differ between LSR and OSR during the 30-day to 6-month followup period. Consideration of total postoperative morbidity shows a $27 \%$ reduction in major morbidity for patients undergoing laparoscopic surgery for diverticular disease.

Keywords Diverticular disease - Diverticulitis - Sigmoid resection - Laparoscopic

Laparoscopic surgery for the treatment of diverticular disease has gained popularity over the past two decades. Particularly in an elective setting, laparoscopic sigmoid resections (LSRs) have shown beneficial effects on postoperative outcomes [1, 2]. Traditionally, elective open sigmoid resections (OSRs) have been associated with high postoperative complication rates and a mortality rate of 
2-5\% [3]. Furthermore, new insights into the natural history of diverticular disease have shown that once it is treated conservatively for an uncomplicated episode, recurrent disease will follow a rather mild course $[4,5]$. Together, these two aspects have resulted in a more conservative policy for the treatment of diverticular disease [6].

In contrast, the reported short-term morbidity and mortality rates for laparoscopic resections are substantially lower than for the open resections [1, 2, 7]. Moreover, the incidence of diverticular disease is growing, primarily among younger people [8]. These findings have revived the discussion on elective resections for diverticular disease. A more aggressive approach again has already been proposed [9]. Data on late outcomes after LSR versus OSR are scarce but could be of decisive value in this ongoing controversy.

In January 2009, the short-term results of the Sigma trial comparing laparoscopic LSR and OSR were published [10]. This prospective, multicenter, randomized control trial showed a significant $15.4 \%$ reduction in major complication rates, less pain, shorter hospital stays, and improved quality of life at the cost of a longer operating time with the laparoscopic approach. Current data complement these 6-month follow-up outcomes comparing the midterm effects of LSR and OSR for patients with symptomatic diverticulitis.

\section{Patients and methods}

The short-term results of the Sigma trial regarding perioperative parameters and postoperative recovery up to 6 weeks after surgery have been published previously. The methodologic and operative details can be found in this report and the earlier published protocol $[10,11]$.

The Sigma trial was a prospective, multicenter, doubleblind, parallel-arm, randomized control trial. In this trial, 104 patients with symptomatic diverticulitis of the sigmoid colon were randomized to undergo either OSR or LSR. The patients and the hospital staff were blinded to the allocation sequence during the initial admission. The study was conducted in accordance with the principles of the Declaration of Helsinki and good clinical practice guidelines. The study protocol was approved by the institutional review boards of all the participating institutions. Before randomization, written informed consent was obtained from all the patients.

After discharge, the patients attended the outpatient clinic 6 weeks and 6 months after surgery. Physical examination was performed, and quality-of-life questionnaires were completed. In case of readmission, medical records were prospectively evaluated.

The primary end points of this 6 months follow-up assessment of the trial were mortality, defined as death due to whatever cause occurring from 30 days to 6 months postoperatively, and late complications occurring from 30 days to 6 months postoperatively including incisional hernias, small bowel obstruction, recurrent episodes of diverticulitis, fistula, anastomotic strictures, abscesses, and reoperations.

The secondary outcome measures included (1) a qualityof-life assessment by the Short Form-36 (SF-36) questionnaire [12] preoperatively, then 6 weeks and 6 months after surgery, and (2) restoration of bowel continuity after stoma formation.

\section{Results}

The 104 consecutive patients who underwent elective surgery for symptomatic diverticulitis of the sigmoid colon were randomized in five centers from February 2002 to December 2006. All the patients underwent the allocated intervention. The 52 LSR patients were comparable with the 52 OSR patients in terms of gender, age, body mass index (BMI), American Society of Anesthesiology (ASA) classification, prevalence of comorbid conditions, previous abdominal surgery, preoperative workup, and indication for surgery.

The short-term results of the Sigma trial have been published previously. In summary, LSR was associated with a $15.4 \%$ reduction in major complication rates, less pain, a shorter hospital stay, and improved quality of life at the cost of a longer operating time.

The demographics of the patients with adverse events during the 6-month follow-up period are shown in Table 2. There were no significant differences between LSR and OSR in terms of morbidity and mortality at 6 months postoperatively (Tables 1,3). Two patients in the LSR group died during the follow-up period $(p=0.153)$, leading to an overall mortality rate of $3 \%$. Both cardiac deaths involved patients with no complications.

A total of 7 late complications were recorded in the LSR group versus 12 in the OSR group ( $p=0.205)$. Of these 12 patients, $9(47 \%)$ finally underwent reoperation (4 LSR vs. 5 OSR; $p=0.727$ ) (Table 4). All three incisional hernias were repaired, and one of five patients with a small bowel obstruction underwent adhesiolysis. One patient with a recurrent episode of perforated diverticulitis required a Hartmann's procedure. Three of four enterocutaneous fistulas were taken down: two with a small bowel resection and one without resection. Another patient required a reoperation for removal of retained gauze $(10 \times 10 \mathrm{~cm})$ 4 months after the initial surgery.

It usually is thought that short-term postoperative complications predict a more complicated long-term course. Our series showed no differences in the number of late complications experienced by patients with or without a short-term complication ( 5 vs. $14 ; p=0.251$ ). An exception was the 
Table 1 Mortality

\begin{tabular}{|c|c|c|c|c|}
\hline & Cause of death & $\begin{array}{l}\mathrm{LSR}(n=52) \\
n(\%)\end{array}$ & $\begin{array}{l}\text { OSR }(n=52) \\
n(\%)\end{array}$ & $p$ Value \\
\hline Up to postoperative day 30 & Myocardial infarction $^{\mathrm{a}}$ & $0(0.0)$ & $1(1.9)$ & 0.558 \\
\hline During 30 - to 180 -day follow-up & Heart disease & $2(3.8)^{b}$ & $0(0.0)$ & 0.153 \\
\hline
\end{tabular}

The distribution of dichotomous data are given as percentages. Pearson's chi-square test was used for discrete variables

LSR laparoscopic sigmoid resection, OSR open sigmoid resection

${ }^{a}$ Patient died during hospitalization

b Neither patient had no complications during hospitalization and died outside the hospital

Table 2 Demographics of patients with adverse events during postoperative days 30-180

\begin{tabular}{llll}
\hline & $\begin{array}{l}\text { LSR }(n=7 / 52)^{\mathrm{a}} \\
n(\%)\end{array}$ & $\begin{array}{l}\text { OSR }(n=12 / 52)^{\mathrm{a}} \\
n(\%)\end{array}$ & $p$ Value \\
\hline Male gender & $3(42.9)$ & $3(25.0)$ & 0.419 \\
Age: years (range) & $66(35-75)$ & $69(41-80)$ & 0.571 \\
BMI: kg/m (range) & $27.3(24.1-31.7)$ & $28.5(23-39)$ & 0.409 \\
ASA grade & & & \\
1 & $4(57.1)$ & $5(41.7)$ & 0.652 \\
2 & $3(42.9)$ & $6(50.0)$ & \\
3 & $0(0.0)$ & $1(8.3)$ & 0.311 \\
Comorbidity & $3(42.9)$ & $8(66.7)$ & \\
Cardiac & 1 & 4 & \\
Crohn's disease & 1 & 0 & 0.539 \\
Diabetes mellitus & 1 & 1 & \\
Hypertension & 0 & 3 & 0.017 \\
Previous abdominal surgery & $5(71.4)$ & $10(83.3)$ & 0.017 \\
Indication for initial sigmoid resection & & & 0.211 \\
Recurrent diverticulitis & $2(28.6)$ & $10(83.3)$ & \\
Sigmoid stricture & $5(71.4)$ & $2(16.7)$ & $2(16.7)$ \\
Major complications up to postoperative day 30 & $3(42.9)$ & 2 & 0 \\
Anastomotic leakage & 2 & 1 &
\end{tabular}

occurrence of enterocutaneous fistulas, which was significantly associated with short-term abscesses or anastomotic leakages $(75 \% ; p=0.002)$. Moreover, consideration of total postoperative morbidity (follow-up period, 0-6 months) showed that 32 patients $(31 \%)$ experienced one or more complications during this follow-up period. Table 5 presents the total postoperative major morbidity (follow-up period, 0-6 months) per study arm. A significant $27 \%$ reduction in major morbidity by the laparoscopic approach can be observed (9 LSR vs. 23 OSR; $p=0.003$ ).

An ostomy was created for 11 patients (6 LSR vs. 5 OSR; $p=0.750)$. Three protective ileostomies were created at the initial operation, and eight resulted from anastomotic leakages followed by Hartmann's procedure (Table 6). One of the patients required a Hartmann's procedure although a protective ileostomy already had been created. For only $3(27 \%)$ of 11 patients with a temporary ostomy was bowel continuity actually restored.
The SF-36 data are depicted in Fig. 1. Scale scores of physical functioning, role limitations due to physical health, role limitations due to emotional problems, energy/ fatigue, emotional well-being, social functioning, pain, general health, and health change were measured preoperatively, then 6 weeks and 6 months postoperatively. There were no preoperative intergroup differences. At 6 weeks postoperatively, the LSR patients had significantly better SF-36 data for role limitations due to physical and emotional problems, social functioning, and pain level. At the 6-month follow-up evaluation, these differences could no longer be demonstrated.

\section{Discussion}

The published short-term data of the Sigma-trial have shown a reduction in major complication rates, less pain, a 
Table 3 Indications for readmissions during days 30-180

\begin{tabular}{|c|c|c|c|}
\hline & $\begin{array}{l}\text { LSR } \\
(n=52) \\
n(\%)\end{array}$ & $\begin{array}{l}\text { OSR } \\
(n=52) \\
n(\%)\end{array}$ & $p$ Value \\
\hline Morbidity during days $30-180$ & $7(13.5)$ & $12(23.1)$ & 0.205 \\
\hline Anastomotic stricture & $1(1.9)$ & $1(1.9)$ & 1 \\
\hline Enterocutaneous fistula $^{a}$ & $2(3.8)$ & $2(3.8)$ & 1 \\
\hline Incisional hernia & $1(1.9)$ & $2(3.8)$ & 0.558 \\
\hline Intraabdominal abscess & $1(1.9)$ & $0(0)$ & 0.315 \\
\hline Recurrent diverticulitis ${ }^{\mathrm{b}}$ & $1(1.9)$ & $2(3.8)$ & 0.558 \\
\hline Retained gauze & $0(0)$ & $1(1.9)$ & 0.315 \\
\hline Small bowel obstruction ${ }^{\mathrm{c}}$ & $1(1.9)$ & $4(7.7)$ & 0.169 \\
\hline
\end{tabular}

Morbidity is reported per patient

The distribution of dichotomous data are given as percentages. Pearson's chi-square test was used for discrete variables

LSR laparoscopic sigmoid resection, OSR open sigmoid resection

${ }^{\text {a }}$ One OSR patient with enterocutaneous fistula recovered without surgery

${ }^{b}$ One patient in each arm with recurrent diverticulitis recovered without surgery

${ }^{c}$ One OSR patient required surgery

shorter hospital stay, and improved quality of life at the cost of a longer operating time with the laparoscopic approach [10]. The current data include the 6-month follow-up assessment for the LSR and OSR patients. No differences between the two groups were found in late complications such as incisional hernias, anastomotic strictures, enterocutaneous fistulas, small bowel obstruction due to adhesions, or recurrent episodes of diverticulitis. Nor did the two groups differ in their surgical treatment.
Table 5 Total postoperative morbidity

\begin{tabular}{llll}
\hline & $\begin{array}{l}\text { LSR }(n=52) \\
n(\%)\end{array}$ & $\begin{array}{l}\text { OSR }(n=52) \\
n(\%)\end{array}$ & $p$ Value \\
\hline Morbidity days 0-30 & $5(10)$ & $13(25)$ & 0.038 \\
Morbidity days 30-180 & $7(14)$ & $12(23)$ & 0.205 \\
Morbidity days 0-180 & $9(17)$ & $23(44)$ & 0.003 \\
\hline
\end{tabular}

The distribution of dichotomous data are given as percentages. Pearson's chi-square test was used for discrete variables. Morbidity is reported per patient. Three LSR patients and two OSR patients had more than one complication

$L S R$ laparoscopic sigmoid resection, $O S R$ open sigmoid resection

The improved quality of life 6 weeks postoperatively returned to baseline values 6 months after surgery. Consideration of total postoperative morbidity (follow-up period, 0-6 months) shows that the laparoscopic approach results in a significant $27 \%$ reduction in major complications.

These midterm results describe a follow-up period of 6 months, as stated in the Sigma trial protocol [11]. Some complications and recurrent episodes of diverticulitis may occur later than 6 months postoperatively. Therefore, a long-term evaluation will be performed at the proper time. Late complications yet to be expected may be related to the approach (incisional hernias or intestinal obstruction), the operative technique (anastomotic stenosis), or the disease itself (recurrence). Retrospective data on these long-term outcomes are rare, and no other randomized control trials on elective surgery for diverticular disease have been reported.

In this study, 11 ostomies were created ( 3 provided as a protective ileostomy at the initial operation and 8 resulting

Table 4 Treatment for morbidity during days $30-180$

\begin{tabular}{llll}
\hline & $\begin{array}{l}\text { LSR }(n=7 / 52)^{\mathrm{a}} \\
n(\%)\end{array}$ & $\begin{array}{l}\text { OSR }(n=12 / 52)^{\mathrm{a}} \\
n(\%)\end{array}$ \\
\hline Reoperations & $4(57.1)$ & $5(41.7)$ & 0.727 \\
$\quad$ Adhesiolysis & $0(0.0)$ & $1(1.9)$ & 0.315 \\
Enterocutaneous fistula takedown & $2(3.8)$ & $1(1.9)$ & 0.558 \\
Hartmann's procedure for perforated diverticulitis & $1(1.9)$ & $0(0.0)$ & 0.315 \\
Incisional hernia repair & $1(1.9)$ & $2(3.8)$ & 0.558 \\
Removal of retained gauze & $0(0.0)$ & $1(1.9)$ & 0.315 \\
Endoscopic dilation of anastomosis & $1(14.3)$ & $0(0)$ & 0.683 \\
Percutaneous drainage of abscess & $1(14.3)$ & $6(50.0)$ & 0.315 \\
Conservative management & $1(14.3)^{\mathrm{b}}$ & 0.361 \\
\hline
\end{tabular}

The distribution of dichotomous data are given as percentages. Pearson's chi-square test was used for discrete variables. Reinterventions and complications are reported per patient

LSR laparoscopic sigmoid resection, OSR open sigmoid resection

${ }^{\text {a }}$ Of the 52 patients, 7 were originally randomized to LSR and 12 to OSR

b See footnote in Table 3 for details 
Table 6 Creation and reversal of ostomies

\begin{tabular}{|c|c|c|c|}
\hline & $\begin{array}{l}\operatorname{LSR}(n=6 / 52)^{\mathrm{a}} \\
n(\%)\end{array}$ & $\begin{array}{l}\text { OSR }(n=5 / 52)^{\mathrm{a}} \\
n(\%)\end{array}$ & $p$ Value \\
\hline Total number of ostomies ${ }^{b}$ & $6(11.5)$ & $5(9.6)$ & 0.750 \\
\hline Hartmann's procedure & $5(9.6)^{\mathrm{c}}$ & $4(7.7)$ & 0.727 \\
\hline Protective loop ileostomy & $2(3.8)$ & $1(1.9)$ & 0.558 \\
\hline Reversal of ostomy & $2(33.3)$ & $1(20.0)$ & 0.558 \\
\hline After Hartmann's procedure & $1(16.7)$ & $1(20.0)$ & 1 \\
\hline After loop ileostomy & $1(16.7)$ & $0(0.0)$ & 0.315 \\
\hline
\end{tabular}

The distribution of dichotomous data are given as percentages. Pearson's chi-square test was used for discrete variables

LSR laparoscopic sigmoid resection, OSR open sigmoid resection

${ }^{\text {a }}$ Of the 52 patients, 6 were originally randomized to LSR and 5 to OSR

${ }^{\mathrm{b}}$ During the initial admission within 30 days after surgery, 10 ostomies were created

c One patient with a protective loop ileostomy also required Hartmann's procedure
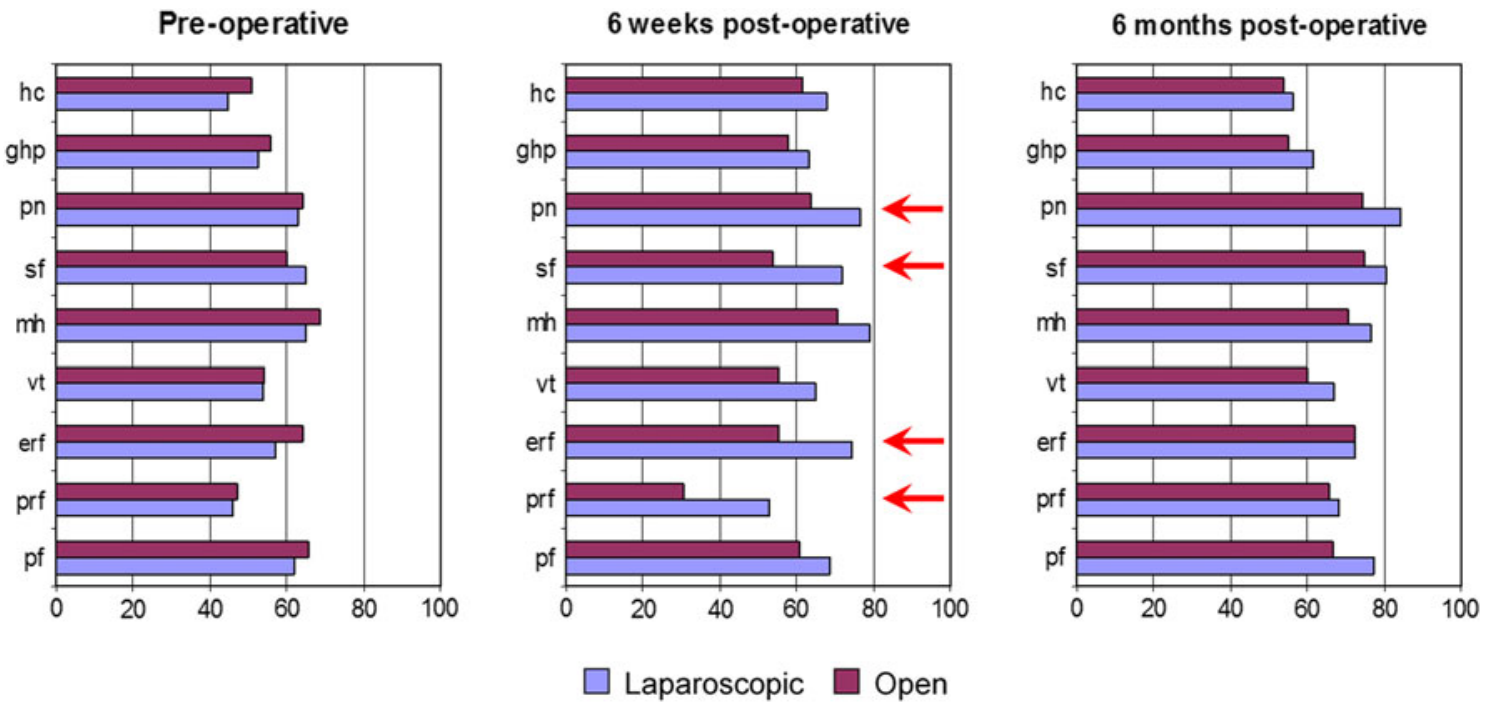

Fig. 1 Mean scale scores of the Short Form-36 (SF-36) questionnaire. The arrows indicate significantly different data between laparoscopic sigmoid resection (LSR) and open sigmoid resection (OSR) for PRF $(p=0.039)$, ERF $(p=0.024), \mathrm{SF}(p=0.015)$, and $\mathrm{PN}(p=0.032)$. A higher score indicates a better outcome [10]. PRF

from anastomotic leakage after Hartmann's procedure). One patient still had a clinically important anastomotic leakage despite creation of a protective ileostomy. Remarkably, only three (30\%) of these temporary ostomies were closed. This number seems low compared with the 90\% reversal after Hartmann's procedure presented by Oomen et al. [13]. Other series present reversal rates of $31-85 \%[14,15]$. The primary difference is that the eight Hartmann's procedures in this study all followed anastomotic leakages and not a primary peritonitis in the case of Hinchey stages 3 and 4 diverticulitis. It may be that such a severely complicated course makes patients more apprehensive to undergo another elective procedure. role limitations due to physical health, $E R F$ role limitations due to emotional problems, $S F$ social functioning, $P N$ pain, $P F$ physical functioning, $V T$ energy/fatigue, $M H$ emotional well-being, $G H P$ general health, $H C$ health change

The quality-of-life assessment showed significantly improved role limitations due to physical health as well as role limitations due to emotional problems, social functioning, and pain 6 weeks after surgery. Analysis of this questionnaire at the 6-month follow-up evaluation showed no significant advantages of LSR. This may be explained by the substantial drop in the response rate, with $27 \%$ of patients not completing the 6-month questionnaire. The short-term improvements in several aspects of the qualityof-life assessment are in accord with the reduced complication rate and shorter hospital stay. After 6 months, these inconveniences seem to be resolved, which may be linked to a similar long-term complication risk in both groups. 
In conclusion, the laparoscopic approach offers shortterm advantages over the open approach for elective sigmoid resections used to manage diverticular disease. Comparable outcomes were found during the 6-week and 6-month follow-up periods in terms of late complications, mortality, and quality of life. For patients presenting with symptomatic diverticular disease of the sigmoid colon, LSR should be preferred over OSR because it offers achievement of a $27 \%$ reduction in total postoperative morbidity.

Disclosures Bastiaan R. Klarenbeek, Roberto Bergamaschi, Alexander A. F. A. Veenhof, Donald L. van der Peet, Wim T. van den Broek, Elly S. M. de Lange, Willem A. Bemelman, Pieter Heres, Antonio M. Lacy, and Miguel A. Cuesta have no conflicts of interest or financial ties to disclose.

\section{References}

1. Alves A, Panis Y, Slim K, Heyd B, Kwiatkowski F, Mantion G (2005) French multicentre prospective observational study of laparoscopic versus open colectomy for sigmoid diverticular disease. Br J Surg 92:1520-1525

2. Schwandner O, Farke S, Fischer F, Eckmann C, Schiedeck TH, Bruch HP (2004) Laparoscopic colectomy for recurrent and complicated diverticulitis: a prospective study of 396 patients. Langenbecks Arch Surg 389:97-103

3. Oomen JL, Engel AF, Cuesta MA (2006) Outcome of elective primary surgery for diverticular disease of the sigmoid colon: a risk analysis based on the POSSUM scoring system. Colorectal Dis 8:91-97

4. Chapman J, Davies M, Wolff B, Dozois E, Tessier D, Harrington J, Larson D (2005) Complicated diverticulitis: is it time to rethink the rules? Ann Surg 242:576-581

5. Salem TA, Molloy RG, O'Dwyer PJ (2007) Prospective, fiveyear follow-up study of patients with symptomatic uncomplicated diverticular disease. Dis Colon Rectum 50:1460-1464

6. Rafferty J, Shellito P, Hyman NH, Buie WD (2006) Practice parameters for sigmoid diverticulitis. Dis Colon Rectum 49:939-944
7. Scheidbach H, Schneider C, Rose J, Konradt J, Gross E, Barlehner E, Pross M, Schmidt U, Kockerling F, Lippert H (2004) Laparoscopic approach to treatment of sigmoid diverticulitis: changes in the spectrum of indications and results of a prospective, multicenter study on 1,545 patients. Dis Colon Rectum 47:1883-1888

8. Etzioni DA, Mack TM, Beart RW Jr, Kaiser AM (2009) Diverticulitis in the United States: 1998-2005: changing patterns of disease and treatment. Ann Surg 249:210-217

9. Jones OM, Stevenson AR, Clark D, Stitz RW, Lumley JW (2008) Laparoscopic resection for diverticular disease: follow-up of 500 consecutive patients. Ann Surg 248:1092-1097

10. Klarenbeek BR, Veenhof AA, Bergamaschi R, van der Peet DL, van den Broek WT, de Lange ES, Bemelman WA, Heres P, Lacy AM, Engel AF, Cuesta MA (2009) Laparoscopic sigmoid resection for diverticulitis decreases major morbidity rates: a randomized control trial: short-term results of the Sigma trial. Ann Surg 249:39-44

11. Klarenbeek BR, Veenhof AA, de Lange ES, Bemelman WA, Bergamaschi R, Heres P, Lacy AM, van de Broek WT, van der Peet DL, Cuesta MA (2007) The Sigma trial protocol: a prospective double-blind multicentre comparison of laparoscopic versus open elective sigmoid resection in patients with symptomatic diverticulitis. BMC Surg 7:16

12. Aaronson NK, Muller M, Cohen PD, Essink-Bot ML, Fekkes M, Sanderman R, Spranger MA, te Velde A, Verrips E (1998) Translation, validation, and norming of the Dutch language version of the SF-36 Health Survey in community and chronic disease populations. J Clin Epidemiol 51:1055-1068

13. Oomen JL, Cuesta MA, Engel AF (2005) Reversal of Hartmann's procedure after surgery for complications of diverticular disease of the sigmoid colon is safe and possible in most patients. Dig Surg 22:419-425

14. Collins D, Winter DC (2008) Elective resection for diverticular disease: an evidence-based review. World J Surg 32:2429-2433

15. Wong WD, Wexner SD, Lowry A, Vernava A III, Burnstein M, Denstman F, Fazio V, Kerner B, Moore R, Oliver G, Peters W, Ross T, Senatore P, Simmang C (2000) Practice parameters for the treatment of sigmoid diverticulitis-supporting documentation. The Standards Task Force. The American Society of Colon and Rectal Surgeons. Dis Colon Rectum 43:290-297 